\title{
Fibrosarcoma developing in the Parotid Gland: An Unusual Presentation
}

\author{
${ }^{1}$ Shagufta Qadri, ${ }^{2}$ Nishat Afroz, ${ }^{3}$ Divya Rabindranath, ${ }^{4}$ Shakeba Qadri
}

\begin{abstract}
Primary sarcomas of the salivary glands occur rarely with few cases having been reported. Fibrosarcoma is a rare type of primary sarcoma with a poor prognosis and low life expectancy. Microscopically, these tumors resemble their counterparts encountered in other areas. They can be easily confused with spindle cell (sarcomatoid) carcinomas, myoepithelial carcinomas or malignant melanomas. We present an unusual case of a primary fibrosarcoma that arose in the right parotid gland of a 50 years male, who presented with a large nodular mass in the right preauricular region. Computed tomography (CT) scan revealed a tumor arising from the right parotid gland. A near total parotidectomy was performed and the histopathology report showed neoplastic spindle cells predominantly arranged in a 'herring bone' pattern. On immunohistochemistry, the tumor was positive for vimentin and was negative for pancytokeratin and S-100, which lead to a firm diagnosis of fibrosarcoma.
\end{abstract}

Keywords: Fibrosarcoma, Parotid gland, Salivary gland, Sarcoma.

How to cite this article: Qadri S, Afroz N, Rabindranath D, Qadri S. Fibrosarcoma developing in the Parotid Gland: An Unusual Presentation. Int J Head Neck Surg 2015;6(2):99-102.

\section{Source of support: Nil}

Conflict of interest: None

\section{INTRODUCTION}

Parotid malignancies represent 1 to $3 \%$ of all head and neck cancers, ${ }^{1}$ while sarcomas comprise only 0.3 to $1.5 \%$ of all major salivary gland neoplasms. ${ }^{2}$ The most frequently described sarcomas of the parotid gland are rhabdomyosarcoma and malignant fibrous histiocytoma (MFH), although less commonly a variety of other tumors can occur, including leiomyosarcoma, malignant peripheral nerve sheath tumor (MPNST), angiosarcoma, osteosarcoma, fibrosarcoma, liposarcoma, hemangiopericytoma, Kaposi sarcoma synovial sarcoma, and alveolar

\footnotetext{
${ }^{1}$ Senior Resident, ${ }^{2}$ Professor, ${ }^{3,4}$ Resident

${ }^{1-3}$ Department of Pathology, Jawaharlal Nehru Medical College Aligarh, Uttar Pradesh, India

${ }^{4}$ Department of Maxillofacial Surgery, Sir Ziauddin Dental College Aligarh, Uttar Pradesh, India

Corresponding Author: Shagufta Qadri, Senior Resident Department of Pathology, Jawaharlal Nehru Medical College Aligarh, Uttar Pradesh, India, Phone: 919897532879, e-mail: qadridrshagufta@yahoo.com
}

soft-part sarcoma. ${ }^{2}$ Fibrosarcoma of the head and neck represents $5 \%$ of all fibrosarcomas. Microscopically, fibrosarcoma shows an intense proliferation of spindle shaped cells, varying a little in their size and shape and arranged in parallel bands which partly cross each other at acute angles (herring bone pattern). ${ }^{3}$ They show a considerable morphological and histopathological overlapping with spindle cell (sarcomatoid) carcinomas, myoepithelial carcinomas and malignant melanomas. Hence, immunohistochemistry is mandatory to differentiate fibrosarcoma from the above-mentioned tumors.

Malignant spindle cell tumors within the salivary gland is a diagnostic challenge for the pathologist. Through this report, the authors are elucidating the existence of fibrosarcoma in the parotid gland and simultaneously emphasizing the need for a high index of suspicion to rule out other lesions with a similar appearance in order to reach the correct diagnosis and form an effective plan of management.

\section{CASE REPORT}

A 50 years Indian male presented to the Outpatient Clinic of the Department of Surgery with a large mass in the right preauricular region for the past 8 months, which progressively increased from a small nodule to the present size. No history of ionizing radiation exposure to the head and neck area could be elicited. The patient consulted a surgeon 5 months previously when the size of the swelling was $3 \times 4 \mathrm{~cm}$. Fine needle aspiration cytology (FNAC) was performed and the smears were suggestive of spindle cell neoplasm. Subsequently, excisional biopsy was advised, but the patient refused to undergo surgery. Local examination revealed a $9 \times 8 \mathrm{~cm}$, fungating mass in the right preauricular region with an irregular, nodular surface, slightly tender to touch, firm to hard in consistency, immobile and fixed to the underlying tissue. Cervical lymphadenopathy was absent and no sign of facial nerve involvement was seen. Fine needle aspiration cytology smears were reviewed and cytology revealed a highly cellular smear with relatively monomorphic spindle-shaped cells with hyperchromatic, fusiform and tapering nuclei with scanty, elongated and poorly delimited cytoplasm enmeshed with stromal element (Fig. 1).

Computed tomography (CT) scan revealed a hypoattenuated mass measuring $9 \times 8 \mathrm{~cm}$, with ill-defined margins 
arising from right parotid gland along with the involvement of bone (Fig. 2).

Patient underwent a near total parotidectomy sparing the facial nerve and the resected specimen was submitted for histopathological examination. Gross examination showed an unencapsulated, nodular mass partially covered with skin showing surface ulceration and fungation. Areas of hemorrhage and necrosis were also noted (Fig. 3). Cut-section revealed greyish-white, firm nodular areas with accompanying foci of hemorrhage and necrosis. Extensive histopathological examination of the tumor, revealed sweeping fascicles of neoplastic spindle cells with moderate degree of pleomorphism that exhibited frequent 'herring bone' pattern and entrapment of few non-neoplastic acini and ducts within the tumor (Fig. 4). The tumor also revealed high mitotic activity $(18 / 10 \mathrm{hpf})$ with frequent atypical forms (Fig. 5). Immunohistochemical analysis showed only vimentin positivity (Fig. 6) while pancytokeratin (Fig. 7) and S-100 (Fig. 8) were negative, that helped in excluding the possibility of

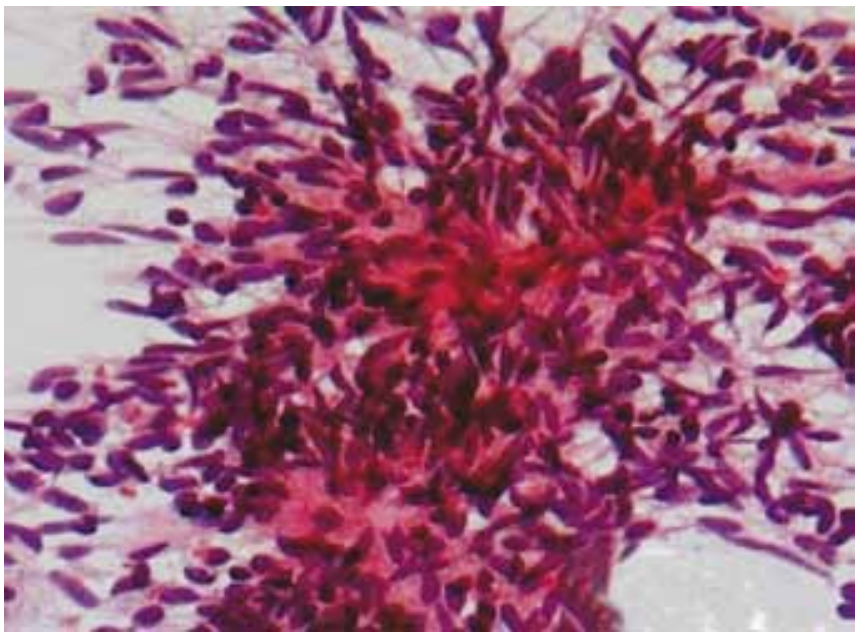

Fig. 1: Fine needle aspiration cytology smear showing high cellularity with monomorphic spindle cell population (H\&E: 400x)

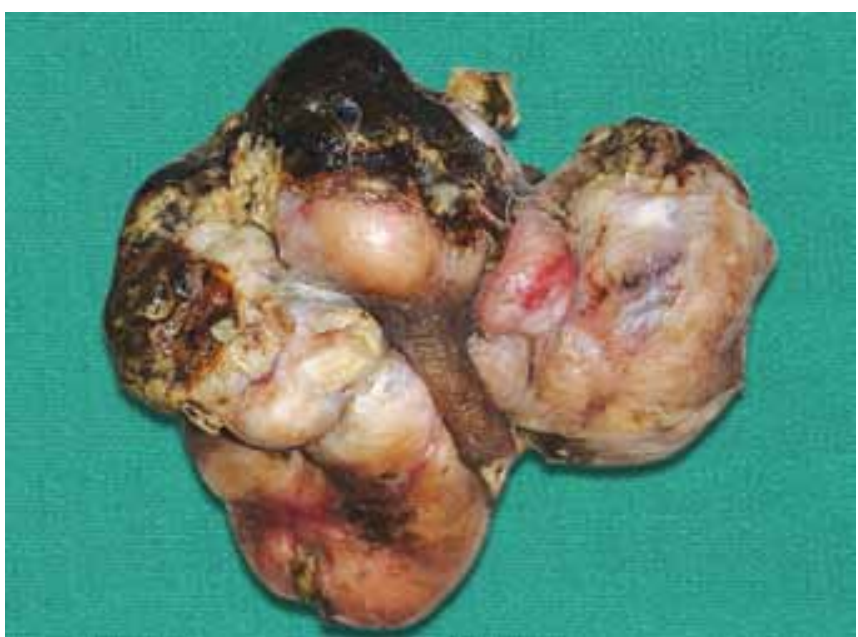

Fig. 3: Gross appearance demonstrating an irregular, nodular, fungating mass, partially covered with skin with surface ulceration carcinosarcoma (malignant mixed tumor) and MPNST respectively, which led to the diagnosis of fibrosarcoma.

Postoperative period was uneventful. Currently, the patient is in follow-up. He is doing well 15 months after surgery.

\section{DISCUSSION}

Tumors of salivary gland originate a diverse group of neoplasms, and they may present significant challenges in the diagnosis as well as in their management. These tumors are rare with an overall incidence in the Western world of approximately 2.5 to 3.0 cases per 100,000 per year. ${ }^{5}$ Malignant salivary gland tumors account for $0.5 \%$ of all malignancies and approximately 3 to $5 \%$ of all head and neck cancers. ${ }^{5,6}$ The most common age of presentation for malignant tumors is in the sixth or seventh decade of life. ${ }^{78}$ Ionizing radiation has been suggested as a cause of salivary gland cancer but the etiology of most salivary gland cancers is still unknown. ${ }^{6,7,9,10}$ Certain occupations are thought to be associated with an increased risk for

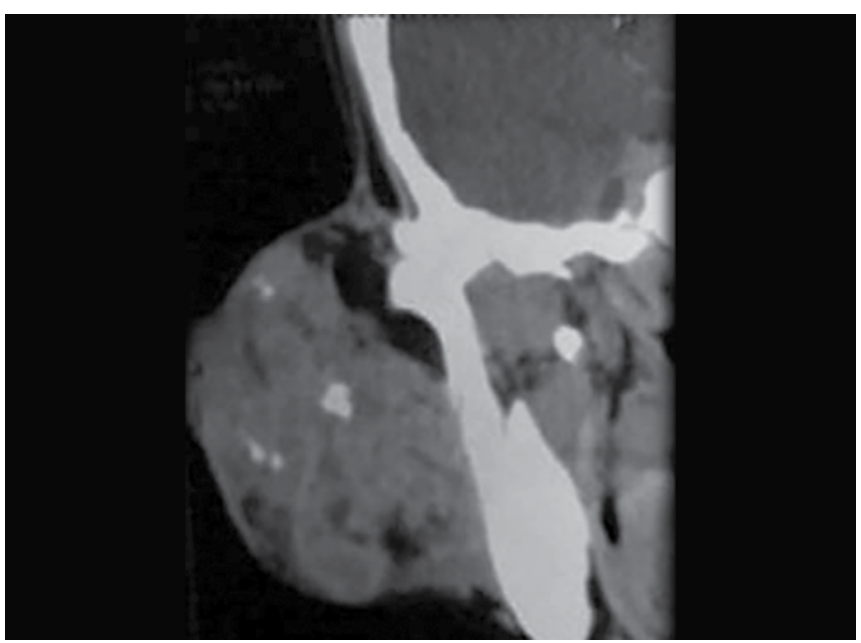

Fig. 2: Computed tomography showing an ill-defined mass protruding from the right parotid gland

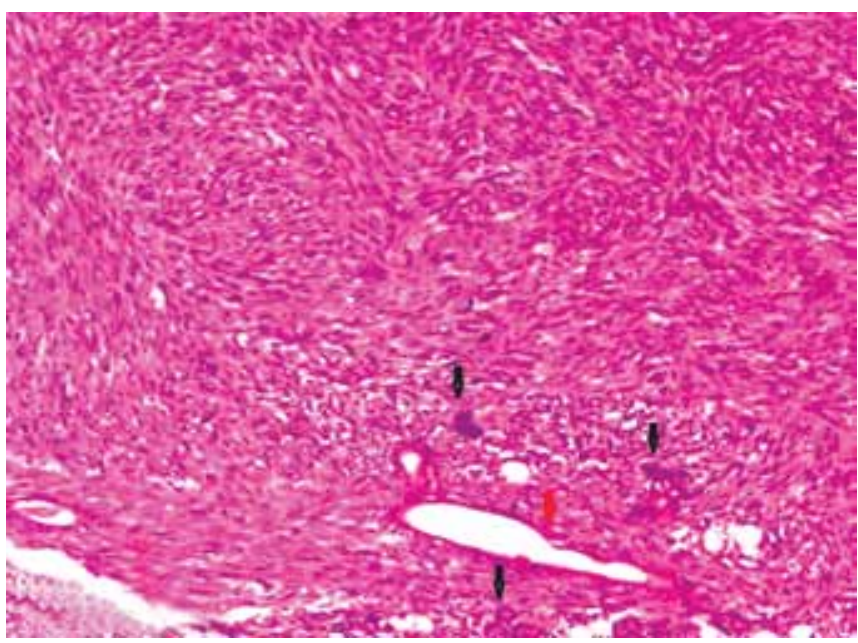

Fig. 4: Sweeping fascicles of mature spindle cells exhibiting frequent herringbone pattern and normal acini and ducts entrapped within the tumor (H\&E: 100x) 


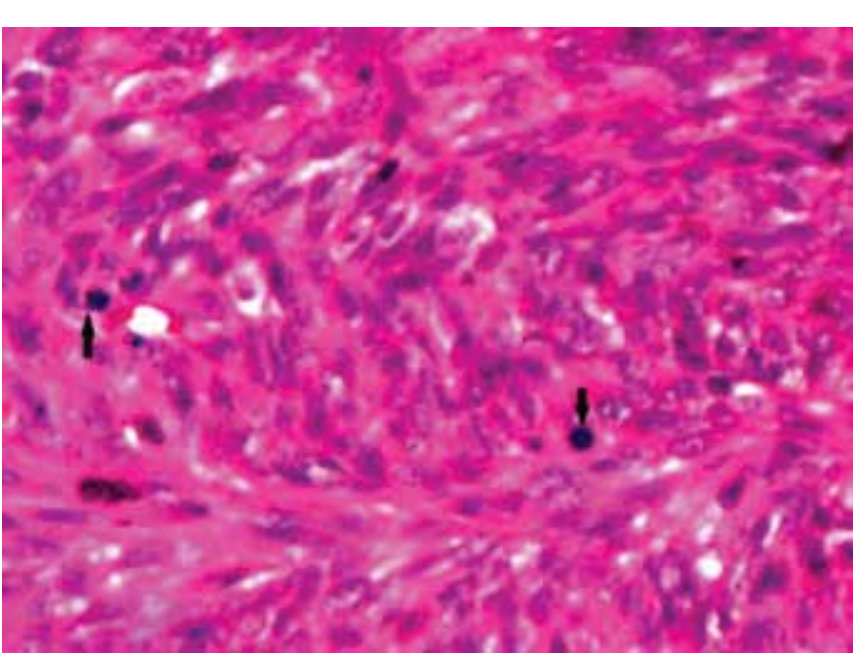

Fig. 5: Spindle-shaped cells with hyperchromatic, fusiform nuclei with scanty, fibrillary cytoplasm and a high mitotic activity (H\&E: 400x)

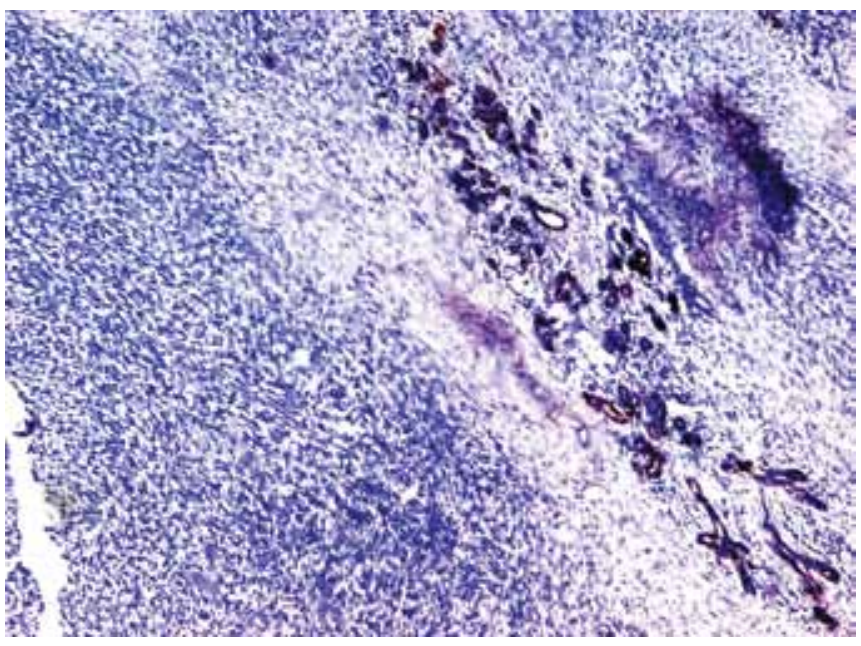

Fig. 7: Tumor cells showing negative immunostaining for pancytokeratin (CK: 100x)

salivary gland cancers including rubber products manufacturing, asbestos mining, plumbing, and some types of wood works. ${ }^{7}$ The majority of the malignant tumors of salivary glands are epithelial in origin, whereas non epithelial tumors constitute less than $5 \%$, of which nearly $90 \%$ are benign. ${ }^{11}$

Primary sarcomas of the parotid are an even rarer group of malignant salivary gland tumors arising from the mesenchymal component of salivary gland and comprises only 0.3 to $1.5 \%$ of all salivary gland neoplasms. ${ }^{2}$ The two most common type of primary sarcomas of the salivary gland have been reported to be malignant schwannoma and fibrosarcoma. ${ }^{4}$ Involvement of the parotid salivary glands are more common than the submandibular glands. ${ }^{4}$ The mean age of occurrence has been reported to be 42 years for men and 38 years for women. ${ }^{4}$ The most common presenting symptom is swelling followed by pain, tenderness or paralysis.

Fibrosarcoma can be the infantile or new adult type. The infantile type occurs inpatients $<2$ years of age with

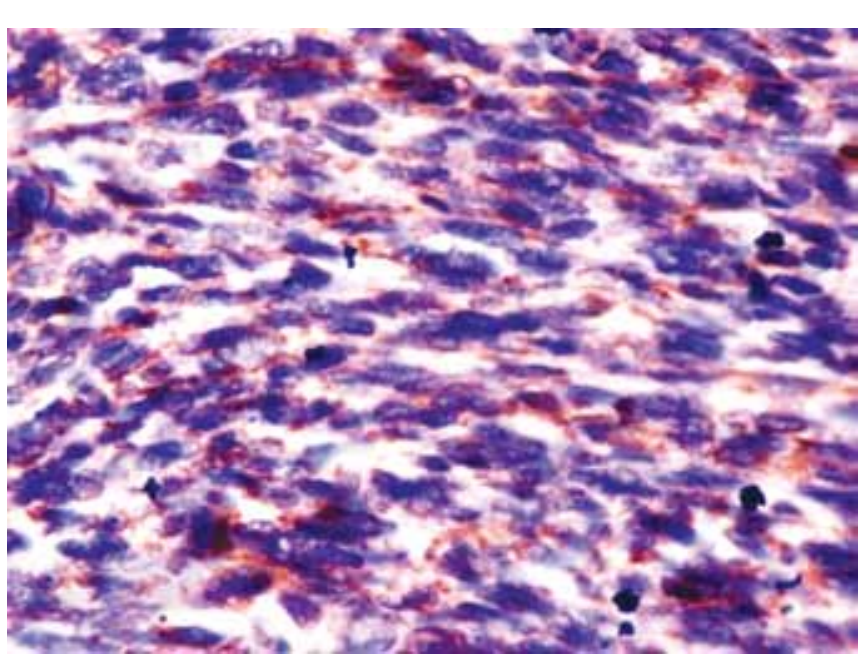

Fig. 6: Vimentin immunostaining showing cytoplasmic positivity in the spindle cells (vimentin: 400x)

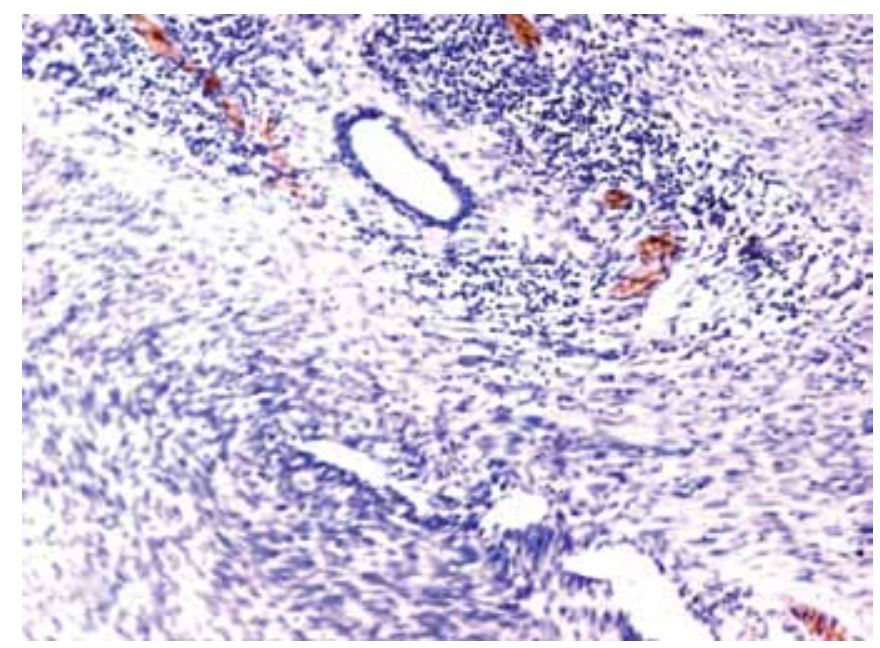

Fig. 8: S100 negativity of the neoplastic spindle cells (S100: 100x)

an intermediate grade of prognosis and rare metastasis, whereas adult fibrosarcoma is a diagnosis of exclusion with a profound metastasizing potential and grave prognosis. Grossly, adult fibrosarcoma appears as a well circumscribed mass with firm white to tan cut surface. Microscopy shows compact spindle cells arranged in short fascicles which split and merge, giving the appearance known as 'herring bone' pattern in a background of variable collagenous stroma. The individual cells are spindle to polygonal in shape with fibrillary eosinophilic cytoplasm and plump hyperchromatic nuclei.

Certain conditions should be ruled out before reaching a diagnosis of fibrosarcoma of the parotid. Firstly, in order to rule out the possibility of metastatic sarcoma, four basic criteria should be met are as follows:

1. There should be no present or past history of a sarcoma elsewhere.

2. Metastasis to the gland from a primary in the skin or mucosa of the upper aerodigestive tract should be excluded. 
3. The overall appearance should be consistent with originating from within the gland, rather than invasion of the gland by a sarcoma of the adjacent soft tissues

4. Multiple sectioning should be done to rule out a carcinosarcoma (so-called true malignant mixed tumor). ${ }^{2}$ Further, the microscopic appearance of the tumor can be easily confused with spindle cell (sarcomatoid) carcinomas, myoepithelial carcinomas, malignant melanomas and MPNST. But the following characteristics helps in limiting the diagnosis to fibrosarcoma, namely:

- Highly cellular spindle cell lesion

- Herring bone arrangement of cells

- Absence of pleomorphism

- S-100 or CK negativity. A study done by Auclair PLet al, highlighted the fact that $15 \%$ of previously diagnosed primary sarcomas of the salivary glands were proven later on to be of epithelial origin. ${ }^{4}$ Immunohistochemistry is, therefore, highly recommended to prevent such diagnostic error.

The most successful therapy is either parotidectomy (superficial or total) or a combination of surgery and radiation. ${ }^{4}$ Wide surgical excision is the mainstay of treatment, although it is also known to be a chemosensitive tumor and as such adjuvant or neoadjuvant chemotherapy is also employed in its management, especially where a wide excision is difficult to achieve. ${ }^{12}$

The prognosis is poor and most of the patients either experience local recurrences or distant metastases and die of the disease. Mean survival time for those dying from the tumor has been reported to be 2.4 years, and a 5 -year survival period remains a significant indicator of cure. ${ }^{4}$

\section{CONCLUSION}

- Fibrosarcoma of the parotid gland is a very rare neoplasm, and it is a diagnosis of exclusion.

- In order to reach a correct diagnosis, metastasis from other body parts or adjacent soft tissue should be ruled out.
- Immunohistochemistry is essential to differentiate these tumors from mixed malignant tumors, melanomas and the tumors myoepithelial origin.

- The delay in diagnosis and treatment should be avoided in order to limit the progression of tumor stage and grade for a better prognosis.

\section{REFERENCES}

1. Kane WJ, Mc Gaffrey TV, Olsen KD, Lewis JE. Primary parotid malignancies. A clinical and pathologic review. Arch Otolaryngol Head Neck Surg 1991 Mar;117(3):307-315.

2. Luna MA, Tortoledo E, Ordonez NG, Frankenthaler RA, Batsakis JG. Primary sarcomas of the major salivary glands. Arch Otolaryngol Head Neck Surg 1991 Mar;117(3):302-306.

3. Soares AB, Lins LH, Macedo AP, Pereira-Neto JS, Vargas PA. Fibrosarcoma originating in the mandible. Med Oral Patol Oral Cir Bucal 2006 May 1;11(3):E243-236.

4. Auclair PL, Langloss JM, Weiss SW, Corio RL. Sarcomas and sarcomatoid neoplasms of the major salivary gland regions. A clinicopathologic and immunohistochemical study of 67 cases and review of the literature. Cancer 1986 Sep 15;58(6):1305-1315.

5. Speight PM, Barrett AW. Salivary gland tumours. Oral Dis 2002;8(5):229-240.

6. Mendenhall WM, Werning JW, Pfister DG. Treatment of head and neck cancer. In: DeVita VT Jr, Lawrence TS, Rosenberg SA. Cancer: principles and practice of oncology. 9th ed. Philadelphia, Pa: Lippincott Williams and Wilkins; 2011. p. 729-780.

7. Ellis GL, Auclair PL. Tumors of the salivary glands. Washington, DC: Armed Forces Institute of Pathology. Atlas of Tumor Pathology; 1996.

8. Wahlberg P, Anderson H, Biörklund A, et al. Carcinoma of the parotid and submandibular glands: a study of survival in 2465 patients. Oral Oncol 2002;38(7):706-713.

9. Scanlon EF, Sener SF. Head and neck neoplasia following irradiation for benign conditions. Head Neck Surg 1981;4(2): 139-145.

10. Van der Laan BF, Baris G, Gregor RT, et al. Radiation-induced tumours of the head and neck. J Laryngol Otol 1995;109(4): 346-349.

11. Bonet M, Zapater E, Alba JR, Basterra J. The acromion thoracic flap: a reconstructive resource after exeresis of a parotid fibrosarcoma. Med Oral Patol Oral Cir Bucal 2011; 16(3):e397-399.

12. Muzumdar D, Michand J, Ventureyre EC. Primary giant congenital infantile fibrosarcoma of the scalp: case report and review of literature. Childs Nerv Syst 2006 Mar;22(3):300-304. 